\title{
LA CONVERTIBILIDAD DE LOS FENÓMENOS Y LA CONSERVACIÓN DE LA ENERGÍA
}

\author{
Ángel Romero*, María Mercedes Ayala, Francisco Malagón*, \\ Edwin García*, Martha Cecilia Gómez**
}

\begin{abstract}
The points of view of Joule and Mayer that led to the Energy Conservation Principle is considered. Their points of view are contrasted with the related to equilibriumnonequilibrium squeme in dealing with the relation between thermical and other phenomena.
\end{abstract}

\section{RESUMEN}

Se analizan los puntos de vista de Joule y Mayer que dan origen al principio de conservación de la energía. Se contraponen estas perspectivas con el planteamiento derivado del esquema equilibración-desequilibración para el análisis de las relaciones entre los fenómenos térmicos y otros fenómenos.

Puede afirmarse que la conservación de la energía hunde sus raíces en el establecimiento y organización de los así llamados principios activos del Universo, teniendo como referencia la convertibilidad de fenómenos. El principio de causalidad, entendido como una forma del entendimiento humano, es el eje de la estructuración de dicha organización y con el término fuerza - considerada como poder y no como acción y que tiene su origen en el principio de causalidad- se suele hacer referencia a tal actividad. En el artículo mostraremos las formas diferentes que adopta el principio de causalidad en los planteamientos de Joule y de Mayer y las connotaciones particulares que adquiere el principio de conservación de la fuerza (o de la energía, en términos más actuales), y la contrastaremos con las implicadas en el planteamiento derivado del esquema equilibración-desequilibración para el análisis de las relaciones entre los fenómenos térmicos y otros fenómenos.

\footnotetext{
Profesores Departamento de Física, Universidad Pedagógica Nacional. e-mail: ayalam@uni.pedagogica.edu.co

* Se sintetizan algunos de los avances de la investigación "La termodinámica fenomenológica: orígenes conceptuales y elementos para una propuesta pedagógica".
} 


\section{PERSPECTIVA DE JOULE}

Para Joule la impenetrabilidad y la extensión más que ser propiedades de la materia son su definición. Tal materia está dotada de propiedades que pueden ser tanto comunes a todos los cuerpos como presentes en forma variable. Dentro de las propiedades de la primera clase se encuentran la gravedad y la inercia, mientras que dentro de las que son variables, y que de paso son las que permiten diferenciar entre cuerpos, se encuentra la llamada fuerza viva o fuerza gastada en poner un cuerpo en movimiento. Esta fuerza -según Joule- puede transmitirse de un cuerpo a otro y es llevada por el cuerpo al cual se transmite, existiendo con él y en él a través de todo el curso de su movimiento.

Existen varias formas de producir o comunicar a un cuerpo fuerza viva, pero dado que, de acuerdo con la experiencia, las formas más comunes de producirla es por medio de la acción gravitacional y de la acción de un resorte deformado, se dice que la fuerza viva es equivalente tanto a la acción de la gravedad como a la acción de la deformación de un resorte a través de una distancia dada.

Teniendo en cuenta estas consideraciones, para Joule la fuerza viva no puede destruirse sin producir su equivalente de atracción gravitacional -o deformación de un resorte - a través de una distancia dada. Es posible que por algún tiempo se le pierda el rastro, pero lo cierto es que se le encuentra de nuevo reproducida; si esto no fuera cierto hace mucho tiempo el universo hubiera muerto y todo se habría detenido.

En este sentido, aunque la fuerza viva aparentemente en algunos procesos se destruye o absorbe, la experiencia muestra que un equivalente es producido el cual, en el transcurso del tiempo, puede de nuevo ser reconvertido en fuerza viva. Tal equivalente es el calor.

El calor es concebido como una forma más de aquel principio activo, equivalente —es decir convertible - a las formas ya conocidas: fuerza viva y atracción a través del espacio. A este respecto, Joule es explicito en considerar que aquella idea que ha prevalecido y según la cual el calor es considerado como una sustancia que posee, al igual que cualquier otra forma de materia, impenetrabilidad y extensión es completamente equivocada; siendo fuerza y materia asumidas como entidades diferentes, resultaría imposible la convertibilidad de una en otra.

Entonces ¿cuál es la naturaleza real del calor? Joule considera que el calor es ya sea fuerza viva o atracción a través del espacio. Considera que toda fuerza o todo poder activo, incluido el calor, se reduce a ello.

"La opinión más difundida ha sido que [el calor] es una sustancia que posee, como todo tipo de materia impenetrabilidad y extensión. Hemos mostrado, sin-embargo, que el calor puede convertirse en fuerza viva o en atracción a través del espacio. Es perfectamente claro, que a menos que la materia pueda convertirse en atracción a través del espacio, que es una demasiado absurda para ser tenida en cuenta por el momento, que la hipótesis del calor como sustancia debe caer.

El calor debe por lo tanto consistir ya sea de fuerza viva o de atracción a través del espacio. En el primer caso podemos concebir que las partículas constituyentes de los cuerpos que han sido calentados estén, ya sea en su totalidad o en parte, en estado de movimiento. En el otro caso podemos suponer que las partículas, debido al proceso de 
calentamiento, han sido desplazadas de tal forma que ejercen atracción a través de un espacio mayor.

Estoy inclinado a creer que en ambas hipótesis son válidas; que en algunos casos, particularmente en el caso del calor sensible, indicado por el termómetro, el calor consistirá en la fuerza viva de las partículas de los cuerpos; mientras que en otros, particularmente en el caso del calor latente, el fenómeno es producido por la mayor separación entre las partículas, que hará que éstas se atraigan a través de un espacio mayor"1.

Es a partir de esta natural convertibilidad entre fuerza viva, calor y atracción a través del espacio que es posible asumir que una misma cantidad de fuerza viva o acción de la gravedad a través del espacio deba ser siempre convertida en la misma cantidad de calor y viceversa; es decir, es posible considerar que existe un equivalente mecánico del calor.

"La atracción de $817 \mathrm{lb}$. a través de un espacio de 1 pie es equivalente a y convertible en la fuerza viva poseída por un cuerpo del mismo peso de $817 \mathrm{lb}$ cuando se mueve con una velocidad de 8 pies/seg y esta fuerza viva es de nuevo convertible en la cantidad de calor que puede incrementar la temperatura de $1 \mathrm{lb}$ de agua en $1^{\circ}$ farenheit.

La regla general es que aunque la fuerza viva es aparentemente destruida ya sea por fricción, presión o cualquier otro medio similar, un equivalente exacto de calor es recuperado. La proposición inversa también es cierta, es decir, que el calor no es disminuido ni absorbido sin la producción de fuerza viva o su atracción equivalente a través del espacio...

Ciertamente los fenómenos de la naturaleza ya sean mecánicos, químicos, o vitales, consisten casi enteramente en una conversión continua de atracción a través del espacio, fuerza viva y calor, unos en otros. Así es como el orden es mantenido en el universo nada es sacado de su orden, nada es nunca perdido, sino que la maquinaria entera, complicada como es, trabaja suave y armoniosa mente"2.

\section{PERSPECTIVA DE R. MAYER}

Para Mayer en el universo existen dos tipos de causas las que, hasta donde la experiencia lo indica, no es posible convertir una en otra: la materia ponderable y las llamadas fuerzas o imponderables. Con el término materia se atribuye a los objetos propiedades bien definidas como peso y volumen; al término fuerza se asocia, por el contrario, lo desconocido, lo hipotético. Por ello es necesario hacer de las fuerzas una teoría tan precisa como la de la materia, es decir, volverlas objeto de inquietud real (hacerlas inteligibles) además de establecer las implicaciones que esto conlleva.

Al ser las fuerzas causas, dos propiedades las caracterizan: ser cuantitativamente indestructibles y cualitativamente convertibles. Estas propiedades se deducen del principio causa igual al efecto $(c=e)$ : Por una parte si el efecto e produce otro efecto $\mathbf{f}$ y éste a su vez otro y así sucesivamente, se tendrá una cadena de causa-efecto en donde ningún

\footnotetext{
${ }^{1}$ JAMES JOULE, "Matter, Living Force, and Heat". En LINDSAY B., Historical development of the concept of energy.

${ }^{2}$ lbid.
} 
miembro puede llegar a anularse. Por otra parte, $c=e$ y $e=f$ deben observarse como diferentes manifestaciones de un mismo objeto.

La forma como Mayer asume el principio de causalidad refleja la necesidad del pensamiento de establecer una conexión dinámica entre las diversas situaciones y fenómenos que se dan en la experiencia, cuando éste rechaza la idea de reducir uno en otro. Así: ningún efecto surge sin ninguna causa, ninguna causa desaparece sin un correspondiente efecto.

En este sentido fuerza para Mayer es una entidad que sólo es posible de ser identificada y definida en relación a la convertibilidad de fenómenos.

Inicialmente la fuerza puede considerarse como aquella entidad que a través de su gasto" produce movimiento. El movimiento de una masa, inicialmente en reposo a una cierta distancia de la superficie de la Tierra y dejada luego libremente, no puede surgir sin el gasto de otra clase de fuerza: es el gasto del levantamiento del peso lo que produce el movimiento de dicho peso. Por estar relacionados como causa y efecto, es que la separación espacial es identificada como una fuerza: fuerza de caída.

Por otra parte, dado que la fuerza como causa de movimiento es una entidad indestructible, el movimiento es igualmente una fuerza.

Ambas formas de la fuerza son identificadas como correspondiendo a un efecto mecánico y si se tiene una situación en la que la fuerza de caída es transformada en movimiento y viceversa, tal efecto mecánico total mantiene siempre un valor constante. Esta ley -afirma Mayer-, que es un caso especial del axioma de indestructibilidad de las fuerza, es conocida como el principio de conservación de la vis-viva.

En innumerables casos, sin embargo, se observa que un movimiento cesa sin producir otro movimiento o levantar un peso; pero dado que una fuerza una vez en existencia no puede ser cero sino que debe reaparecer en otra forma. ¿Qué otra forma debe entonces tomar-se por fuerza?

Mayer es claro en afirmar que sólo la experiencia puede proveer información sobre este punto. Por ejemplo, si se frotan dos platos metálicos uno con otro, por una parte, se hace desaparecer el movimiento y, por otra, se observa la producción de calor. En este sentido, entonces, el calor es igualmente una fuerza, en una forma que se distingue de otras; y como es una fuerza puede transformar-se también en una fuerza mecánica..

Pero ¿cómo establecer una conexión entre estas dos formas de fuerza? De la misma manera que para la solución de las ecuaciones que conectan la fuerza de caída y el movimiento, las magnitudes en consideración deben ser determinadas para un situación definida; para la solución de la ecuación existente entre fuerza de caída y movimiento, por una parte, y calor, por otra, se debe dar respuesta a la cuestión de qué tan alto debe ser levantado sobre la superficie de la tierra un peso definido con el fin de que su fuerza de caída sea equivalente al calentamiento de un peso igual de agua de $0^{\circ} \mathrm{C}$ a $1^{\circ}$.

"Si aquí una transformación de calor en efectos mecánicos es planteada como algo que ocurre, es porque se establece como un hecho, y no como algo a ser explicado. Así, una dada cantidad de hielo se transforma en una correspondiente cantidad de agua: este hecho es simplemente así, y permanece independientemente de las infructuosas especulaciones sobre cómo y por qué. La ciencia real permanece satisfecha con el 
conocimiento positivo y libremente deja a poetas y filósofos la solución de tal interminable acertijo con la ayuda de la fantasía...

La química nos enseña a reconocer los cambios cualitativos que experimenta una materia dada en diferentes condiciones. Provee en cada caso individual la prueba de que en un proceso químico sólo cambia la forma y no la magnitud de la materia dada...Lo que la química representa con respecto a la materia, la física lo tiene que representar en el caso de la fuerza. La única misión de la física es llegar a familiarizarse con la fuerza en sus varias formas e investigar las condiciones que gobiernan sus cambios... Que en un futuro sea posible transmutar los elementos químicos unos en otros, y reducirlos a un menor número de elementos más simples, o aún a una única sustancia fundamental, es más que dudoso. La misma situación sin embargo no ocurre para las causas del movimiento. Se puede probar a priori y confirmar en cualquier lugar que las varias formas de la fuerza pueden transformarse unas en otras. En verdad existe una única fuerza"3.

\section{ESQUEMA EQUILIBRACIÓNDESEQUILIBRACIÓN Y CONSERVACIÓN DE LA ENERGÍA}

Un cambio de estado de cualquier cuerpo, cualquiera que sea, remite a preguntarse por la causa de ese cambio; es así, como se suele hablar de calor para designar la causa del cambio del estado térmico de un cuerpo, de fuerza para designar la causa del cambio del estado de movimiento, de carga para designar la causa del cambio de estado de electrificación. El estado en cada caso está identificado por una cierta magnitud intensiva: temperatura en el caso térmico, velocidad en el caso del movimiento, potencial en el caso eléctrico.

Sin embargo, hay otras maneras de dar cuenta de la causa de tal cambio de estado. Por ejemplo, se piensa que para poder mantener la temperatura de un cuerpo se requiere que el entorno de él esté a la misma temperatura que éste, en el mismo estado térmico. Otra forma es la de inhibir la interacción térmica entre el cuerpo y el medio que lo rodea, en el caso que esto no ocurra. Una última forma sería la de buscar un cierto procedimiento para compensar ese cambio de manera simultánea. Desde este punto de vista, los cambios térmicos ocurren únicamente cuando hay un desequilibrio térmico entre el cuerpo en cuestión y el medio que le rodea. Así el desequilibrio térmico establece la condición de posibilidad de un cambio de temperatura; y la equilibración térmica, a la que se tiende espontáneamente, la causa de dichos cambios ${ }^{4}$.

Esta forma relacional de abordar el cambio de estado térmico de un cuerpo que hace del esquema equilibrio-desequilibrio su fundamento, puede ser igualmente usada para dar cuenta de los cambios de estado de un cuerpo, cualesquiera que estos sean: dos cuerpos pueden interactuar debido a su movimiento siempre y cuando sus velocidades difieran; y lo pueden hacer eléctricamente si sus potenciales son diferentes... Perturbar un sistema significa, entonces, ya sea establecer un desequilibrio entre el sistema y el exterior a éste, o entre una parte del sistema y el resto de él.

\footnotetext{
${ }^{3}$ R. MAYER, "The Conservation of Energy en LINDSAY B., Historical development of the concept of energy.

${ }^{4}$ Véase: AYALA, M. M. et al. "El esquema equilibración-desequilibración y los procesos termodinámicos”. Física y Cultura: Cuadernos sobre Historia y Enseñanza de las Ciencias, No. 4,1998.
} 
Dado que desde esta perspectiva un cambio en el estado de una dada clase de un cuerpo implica un cambio de estado de la misma clase de por lo menos otro cuerpo, tal consideración nos lleva a buscar la forma de conectar un cambio con otro, vía la cuantificación de los mismos. Al tratar de dar solución a este problema, y considerando que la interacción se efectúa entre dos cuerpos, es importante tener en cuenta dos aspectos que están íntimamente relacionados:

1) que el cambio en un cuerpo "induce" el cambio en el otro, o, en otras palabras, que el cambio de estado de uno se hace a expensas del otro, exigencia que surge del principio de causalidad; y 2) que aquello que experimenta el cambio de estado debe ser considerado en el intento de cuantificar dicho cambio.

El primer aspecto nos sugiere la igualdad y oposición de los cambios; nos remite a pensar en una magnitud que caracterice la conexión entre dichos cambios y que de cuenta, de alguna forma, de la "reacomodación" efectuada al llevarse a cabo el proceso de equilibración, siendo los cambios de estado realizados su expresión.

El segundo aspecto, por su parte, nos conduce a introducir una magnitud que identifique los cuerpos con relación al cambio de estado de la clase particular que se está indagando.

En este orden de ideas tenemos, por ejemplo, que cuando dos cuerpos en diferentes estados de movimiento interactúan —un choque-, la medida del cambio de estado de movimiento efectuado en dichos cuerpos no depende únicamente de la variación que experimenta la velocidad, $\Delta \mathbf{v}$, sino que se requiere introducir la masa $\mathbf{m}$ como la magnitud que identifica y diferencia a los cuerpos con relación al cambio de estado de movimiento; pudiéndose de esta manera establecer la igualdad, opuesta, exigida entre los cambios. Es así, como el cambio de estado de movimiento de un cuerpo queda especificado por $\mathbf{m} \Delta \mathbf{v}$, magnitud extensiva que se suele designar por $\Delta \mathbf{P}$; siendo entonces $\Delta \mathbf{P}_{1}=-\boldsymbol{\Delta} \mathbf{P} 2$, y donde $\Delta \mathbf{P}_{1}$; se puede considerar como la cantidad de movimiento que fluye del cuerpo 1 al cuerpo 2. En el caso térmico, el cuerpo queda caracterizado por una magnitud que se suele denominar capacidad calorífica $\mathbf{C}$ del cuerpo; la medida del cambio térmico efectuado en éste queda determinado por $\mathbf{C} \boldsymbol{\Delta} \mathbf{T}$, magnitud que se conoce con el nombre de cantidad de calor, siendo $C_{1} \Delta T_{1}=-C_{2} \Delta T_{2} y{ }^{*} C_{1} A T_{1}{ }^{*}$ la cantidad de calor que fluye del cuerpo a temperatura mayor al cuerpo a temperatura menor durante el proceso de equilibración. Para la determinación de la medida del cambio de estado de cualquier otra clase de un cuerpo, se procedería de manera análoga. Es importante resaltar aquí cómo la medida del cambio de una clase dada se determina estableciendo la conexión entre dos cambios de una misma clase de fenómenos, de una misma cualidad; cambios que se suceden siempre y cuando haya un desequilibrio inicial.

Pero, aquí, nuevamente surge el problema de la causa: ¿de dónde procede ese desequilibrio inicial? ¿cuál es su causa?

1. La experiencia en torno a la convertibilidad de fenómenos pone de manifiesto cómo un cambio de una clase puede generar un cambio de otra. Lo cual exige a nuestro pensamiento, cuando se descartan posturas reduccionistas, conectar cambios de diferentes clases, donde uno puede ser asumido como causa del otro. Establecer tal conexión permite establecer una medida del cambio desde un punto de vista absoluto, en cuanto da respuesta al "gasto" invertido en dicho cambio; que se expresa de manera diferente dependiendo de la clase particular de cambio de estado que se considere. 
2. Ese gasto en la producción de un cambio de una clase es el gasto en la producción de un desequilibrio de la misma clase; gasto que se puede hacer por la tendencia espontánea de la naturaleza a la equilibración. Por lo tanto, el cambio que se concibe como causa es debido a una equilibración, mientras que el efecto siempre implica una desequilibración.

3. Para cada cambio de estado de una clase particular se ha determinado una cierta magnitud como medida de ese cambio: cantidad de movimiento que fluye en la equilibración mecánica, cantidad de calor que fluye en la equilibración térmica, etc. Es de esperar que el gasto invertido para que un cambio dado se efectúe, es decir la medida "absoluta del cambio", este relacionado con esa magnitud que identifica el proceso de equilibración y la diferencia de aquella magnitud intensiva que expresa el desequilibrio inicial $^{5}$.

\section{CONCLUSIONES}

Tanto en Joule como en Mayer, los principios activos del Universo se diferencian de los entes materiales (cuerpos) y se caracterizan igualmente de forma ontológica. Tales principios activos, por consiguiente, existen de forma independiente a los cuerpos.

Para Joule el principio activo (la fuerza) se identifica específicamente con las acciones de tipo mecánico; la fuerza es o bien fuerza viva o atracción a través del espacio, siendo una causa o efecto de la otra.

Mayer por su parte adopta una postura no reduccionista; al asumir: 1) que todo efecto está en potencia de convertir-se en causa, y 2) que si bien ningún efecto surge sin ninguna causa, ninguna causa desaparece sin un correspondiente efecto, establece la unidad, la conservación y la convertibilidad de las fuerzas.

Entre tanto, desde la perspectiva del esquema equilibración-desequilibración, se adopta igualmente una postura no reduccionista y el principio activo deja de ser una entidad que "existe» ontológicamente hablando. El desequilibrio es la condición de posibilidad de cualquier cambio, y la equilibración -a la que el Universo tiende espontáneamente-, la causa de dichos cambios. De otra parte, todo desequilibrio que desaparezca, es decir todo restablecimiento de equilibrio, genera un desequilibrio de alguna clase. $Y$ es en el restablecimiento del equilibrio — sea mecánico, eléctrico, químico, etc., - que es posible convertir un fenómeno en otro.

\footnotetext{
${ }^{5}$ Véase: $\quad$ FALK, G.; HERRMANN, F.; SCHMID, GB. "Energy Forms and Energy Carriers", AM. J. Phys. 51, 1983, 1074-1077.

HERRMANN F. y SCHMID, G. B. “An Urto-Date approach to Physics”, AM. J. Phys. 52, 1984, 794-799.
} 